\title{
The Suitable Timing of Visual Sensing in Error Recovery Using Task Stratification and Error Classification
}

\author{
Akira Nakamura, Kazuyuki Nagata \\ Intelligent Systems Research Institute \\ National Institute of Advanced Industrial Science and Technology (AIST) \\ Central 2, 1-1-1 Umezono, Tsukuba, Ibaraki, 305-8568 Japan \\ Kensuke Harada \\ Robotic Manipulation Research Group \\ Systems Innovation Department \\ Graduate School of Engineering Science, Osaka University \\ 1-3 Machikaneyama, Toyonaka 560-8531, Japan \\ Natsuki Yamanobe \\ Intelligent Systems Research Institute \\ National Institute of Advanced Industrial Science and Technology (AIST) \\ Central 2, 1-1-1 Umezono, Tsukuba, Ibaraki, 305-8568 Japan \\ E-mail:a-nakamura@aist.go.jp,k-nagata@aist.go.jp, \\ harada@sys.es.osaka-u.ac.jp,n-yamanobe@aist.go.jp \\ www.aist.go.jp
}

\begin{abstract}
Judgment of errors for recovery is performed during execution of the system. Ideally, it is desirable for the judgment to be performed at several times. However, in that case, many sensors would be needed and it would lead to disturbing the workflow. Therefore, it is important to be able to judge an error efficiently in the most suitable timing and within a few attempts. This paper describes a method for efficient timing of visual sensing for error recovery.
\end{abstract}

Keywords: error recovery, task stratification, error classification, manipulation, sensing timing

\section{Introduction}

In the manipulation tasks performed by robots involved in industrial production and household chores, error recovery techniques are important since robots need to perform complicated tasks. ${ }^{1-6}$ We have proposed an error recovery method using the concepts of task stratification and error classification. ${ }^{7,8}$

In a robot system with error recovery capability, sensing is performed by using vision systems for the judgment of errors mainly at important points. For the purpose of evading errors as much as possible, it is desirable to perform visual sensing for error judgments at several times. However, it is difficult practically since a large number of sensors would be necessary for the hardware and execution system, and the interruption of the workflow and time limitations are also problems. In this paper, a method to derive efficient timing for visual sensing during error recovery is proposed.

Section 2 shows the significance of visual sensing in error recovery. Classification of visual sensing is 
described in Section 3. Efficient timing for visual sensing is shown in Section 4.

\section{Significance of Visual Sensing in Error Recovery}

The robot tasks targeted for this paper are mainly assembly, disassembly, inspection, and parts replacement in industrial factories and power plants. Tasks such as bed-making, cooking and washing performed by robots assisting humans in daily life are also our target.

We proposed a method of error recovery for a manipulation robot system in Ref. 7 and proposed a new method of error recovery in Ref. 8. These methods are based on the concepts of task stratification and error classification.

The robot tasks that we consider basically consist of sequences of sensing, modeling, planning and execution. We consider the sequences to achieve the completion of the main task using a hierarchy, but the robot system is composed of sequences basically for each layer. After execution of the task, the error judgment is performed. That is to say, after execution of the sequence of sensing, modeling, planning and execution for each task, sensing is performed once again to check for an error. A check of whether the objective of the given task has been accomplished is performed in the top layer. In addition, a check of whether the goals of the sub-tasks have been achieved is performed in each layer. Usually, visual sensing is used for judgment of the error status in all layers. Sensing based on vision is performed for the most part in manufacturing processes and in tasks providing human life assistance in particular. Therefore, we also considered the checking of errors by visual sensing in this paper.

There are various methods of checking errors using data obtained by sensing. For example, there are methods using judgment based on raw data, using geometric models derived from the original data, and so on.

\section{Classification of Visual Sensing}

There are many kinds of visual sensors. In our method, visual sensors from which three-dimensional data can be obtained are needed. Depending on the kind of visual

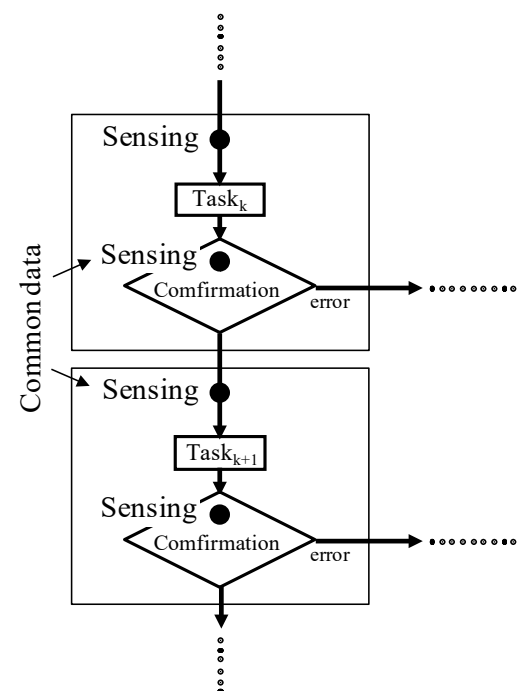

Fig. 1 Common data in successive sub-tasks in (Case 1)

sensor used, the visual data obtained will differ as follows.

(a) Visual sensor from which three-dimensional data can be obtained for moving objects

This is a high-speed three-dimensional data acquisition device such as high-speed stereovision equipment. However, this is not applicable for this paper because such equipment is expensive and unrealistic for general-purpose applications.

(b) Vision sensor for stationary object use only

This is a three-dimensional data acquisition device of the normal-speed stereovision that is inexpensive passive stereovision equipment. The laser range finder of active stereovision is this type, too. For this paper, this is the type of sensor we considered. In addition, a stationary object is ideal for data acquisition. When an object is not stationary, the acquired data may include errors.

\section{Timing of Efficient Visual Sensing}

In this section, we will consider a case in which data is acquired in a stationary state such as (b) of Section 3. In our method, sequences composed of sensing, modeling, planning and execution are performed in each layer 


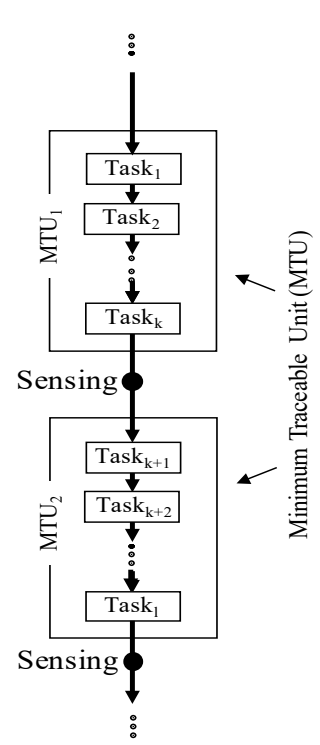

Fig. 2 Sensing points in (Case 2)

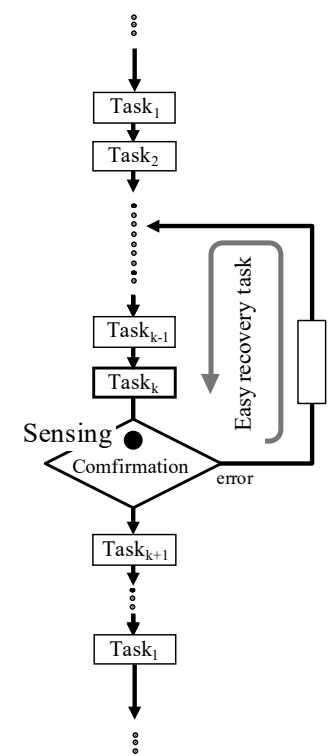

Fig. 3 Sensing point in (Case 3)

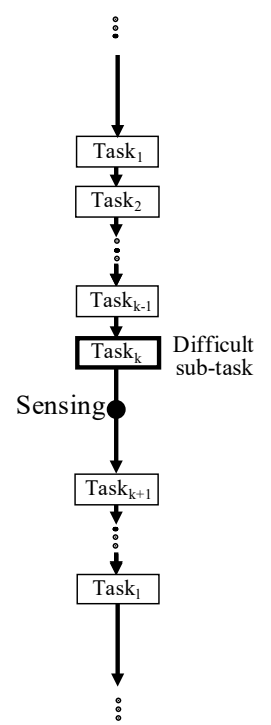

Fig. 4 Sensing point in (Case 4) derived by stratification, but it is difficult to perform sensing to obtain visual data at all sensing points. It is the cause that it takes time, with computational complexity becoming extensive and task motion interruptions increasing. Therefore, it is desirable to keep the timing of data acquisition to a minimum. For this reason, we will explain desirable sensing timing in detail.

(Case 1) Use of sensing data for an error check in the next sub-task

It is sometimes possible that the sensing data acquired for the error check in a sub-task can be used for the data needed first in the next sub-task. If the necessary conditions of both data are the same in the range and precision of the three-dimensional data, they can be used as common data (Fig. 1).

(Case 2) Use of sensing on the basis of a minimum traceable unit

Next, we will show visual sensing on the basis of a minimum traceable unit (MTU), which means the smallest unit in which it is necessary to return to the first node of a motion primitive sequence if an error occurs. Visual sensing is performed just after an MTU, and a check of the error status is performed (Fig. 2).
(Case 3) With consideration for the ease of performing a recovery task

When the place where a recovery task is easy to do is known in advance, a check of whether or not an error has occurred is performed at that point (Fig. 3). When the emphasis is on the ease of the task, this method is effective.

(Case 4) With consideration for the difficulty of accomplishing a sub-task

When the place where a sub-task tends not to achieve the sub-goal or where a failure tends to occur is known in advance, a check of whether or not an error has occurred is performed at that point (Fig. 4). When the emphasis is on the achievement of the sub-task, this method is effective.

(Case 5) With consideration for a change in the range or accuracy of sensing

When the range of sensing varies from the local domain to a wider domain (or the reverse) or when the accuracy of sensing varies from precise to rough (or the reverse), a check of whether or not an error has occurred is performed at that point. For example, local range sensing is performed for manipulator operations and 
global range sensing is performed for transfer of objects by manipulator (Fig. 5). When a camera parameter changes due to the place or the area of the space, this

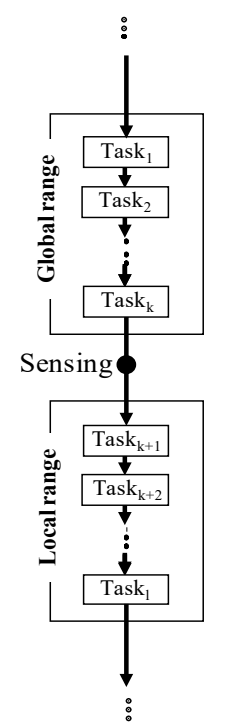

Fig. 5 Sensing point in (Case 5)

method is effective.

We have shown guidelines where the sensor timings can be placed to reduce the number of sensing executions.

\section{Conclusion}

We have shown guidelines for effective timing for visual sensing in a manipulation system with error recovery capability. It is possible to reduce the number of vision sensors and the calculation workload of the software according to the guidelines we suggested. Our future studies will include applicability of this technique to an actual system, derivation of effective sensing timing for a moving manipulation system, error estimation using non-vision sensors, and so on.

\section{Acknowledgement}

This work was supported by JSPS KAKENHI Grant Number 15K00370.

\section{References}

1. L. Seabra Lopes and L. M. Camarinha-Matos, A machine learning approach to error detection and recovery in assembly, in Proc. IEEE/RSJ Int. Conf. on Intell. Robots Syst., (Pennsylvania, USA, 1995), vol. 3, 197-203.

2. T. Fukuda, M. Nakaoka, T. Ueyama and Y. Hasegawa, Direct teaching and error recovery method for assembly task based on a transition process of a constraint condition, in Proc. IEEE Int. Conf. Robot. Autom., (Seoul, Korea, 2001), 1518-1523.

3. K. Yamazaki, M. Tomono, T. Tsubouchi and S. Yuta, Motion planning for a mobile manipulator based on joint motions for error recovery, in Proc. IEEE/RSJ Int. Conf. on Intell. Robots Syst., (Beijing, China, 2006), 7-12.

4. M. Scheutz and J. Kramer, Reflection and reasoning mechanisms for failure detection and recovery in a distributed robotic architecture for complex robots, in Proc. IEEE Int. Conf. Robot. Autom., (Roma, Italy, 2007), 3699-3704.

5. J. C. Himmelstein, E. Ferre and J.-P. Laumond, Teleportation'-Based Motion Planner for Design Error Analys is, in Proc. IEEE Int. Conf. Robot. Autom., (Kobe, Japan, 2009), 914-920.

6. P. Pastor, M. Kalakrishnan, S. Chitta, E. Theodorou and S. Schaal, Skill learning and task outcome prediction for manipulation, in Proc. IEEE Int. Conf. Robot. Autom., (Shanghai, China, 2011), 3828-3834.

7. A. Nakamura and T. Kotoku, Systematization of error recovery in skill-based manipulation, Artificial Life and Robotics, Springer, 14(2) (2009) 203-208.

8. A. Nakamura, K. Nagata, K. Harada, N. Yamanobe, T. Tsuji, T. Foissotte and Y. Kawai, Error recovery using task stratification and error classification for manipulation robots in various fields, in Proc IEEE/RSJ Int. Conf. on Intell. Robots Syst., (Tokyo, Japan, 2013), 3535-3542. 